\title{
Factores que inciden en el crecimiento de las mypes de calzado
}

\section{Factors that affect the growth of shoe mypes}

\author{
Alindor Fernando Espinoza Espinoza \\ https://orcid.org/0000-0001-7391-6173 \\ Carlos Oswaldo Venturo Orbegoso \\ https://orcid.org/0000-0002-7465-8687 \\ Universidad César Vallejo, Perú \\ Oscar Jhonny Bravo Chávez \\ https://orcid.org/0000-0002-2137-5747 \\ Universidad Privada San Juan Bautista, Perú
}

Autor por correspondencia: fer.es20@ hotmail.com; cventuro2911@gmail.com; bravochavez@hotmail.com

Fecha de recepción: 31 de diciembre de 2019 - Fecha de aceptación: 11 de febrero de 2020

\section{Resumen}

El presente trabajo de investigación busca analizar qué factores son los que tienen una mayor incidencia en el crecimiento de las mypes de calzado en la ciudad de Trujillo (Perú), donde el empresario es un elemento importante para crear valor a la empresa. El estudio ha considerado como referencia el marco teórico por Okpara y Wynn (2007), quienes han propuesto unas categorías para mantener un negocio exitoso, ubicados en: operativos, estratégicos, administrativos y externos. La metodología aplicada en el presente trabajo se desarrolla mediante un estudio cualitativo de desarrollo de casos, donde se delimita a doce empresas del sector calzado, que tienen siete criterios para elegir a los entrevistados. Los datos recolectados se obtendrán mediante una guía de entrevistas con sus respectivas categorías, subcategorías e indicadores respectivos para su confiabilidad. Los datos se examinaron mediante el paquete para triangular información denominado Atlas Ti versión 8. Los resultados de la presente investigación muestran la significancia de la categoría factores administrativos, donde se observa que la sub categoría administración general para el empresario, tiene un impacto fuerte en el desarrollo de las mypes de calzado, mientras que la categoría: factores estratégicos, donde la subcategoría infraestructura, no es un factor significativo para su crecimiento.

Palabras clave: factores de éxito; crecimiento; administración; mypes; calzado

\begin{abstract}
This research work seeks to analyze which factors are those that have a greater impact on the growth of shoe mypes in the city of Trujillo, where the entrepreneur is an important element to create value for the company. The study has considered as reference the theoretical framework by Okpara and Wynn (2007), who have proposed some categories to maintain a successful business, located in: operational, strategic, administrative and external. The methodology applied in this work was developed through a qualitative case development study, where twelve companies from the footwear sector were delimited, which had seven criteria to choose the interviewees. The data collected were obtained through an interview guide with their respective
\end{abstract}


categories, subcategories and respective indicators for their reliability. The data was examined using the package to triangulate information called Atlas Ti eight. The results of the present investigation show the significance of the Category: Administrative factors, where it is observed that the General Administration Sub category for the entrepreneur, has a strong impact on the development of footwear mypes, while the category: strategic factors, where the Infrastructure subcategory is not a significant factor for its growth.

Keywords: success factors; growth; administration; mypes; footwear

\section{Introducción}

El sector calzado a nivel internacional es liderado por el continente asiático, integrado por cuatro países como son China, India, Vietnam e Indonesia quienes refieren que la producción de calzado a nivel global se elevó a doce mil millones de pares cada año, representando un $60 \%$ de la exportación mundial. China elabora en promedio 6,500 millones de calzados por año y vende 4 mil millones a otros países. Ciceg (2019) y Urcia (2013) sostuvieron que las grandes industrias de calzado a nivel mundial presentan diversos problemas para mantener su ritmo de crecimiento, bien sea por el precio de los productos, baja innovación, creatividad, diseño, materias primas, poca participación en las ferias y revistas especializadas; tal es el caso que Japón ha descendido su ritmo de crecimiento de calzado en 13.7\% en el periodo 2012 al 2016 (ICEX, 2017).

En Perú, según AMPEX (2018) refiere que la participación de las Mypes en el PBI es de un $24 \%$ en el año 2017 y son alrededor del $96 \%$ del total de las empresas peruanas y el $46.5 \%$ es representada por la población económicamente activa. Para los expertos Serida, Alzamora, Guerrero, Borda, y Morales (2016) sostienen que las normativas vigentes, la transmisión de investigación y desarrollo, y el acceso a la infraestructura tecnológica, así como los costos laborales, representan las condiciones básicas, para el surgimiento de los jóvenes peruanos que se ubican en la industria de calzado. Estos datos conllevan a la necesidad de reconocer aquellos factores que determinan el fortalecimiento de las Mypes de calzado, de tal manera que se pueda avanzar a un mayor número de micro y pequeñas empresas que tiendan a su crecimiento en el estado peruano.

Caruajulca y Fernández (2017) mencionan que el sector calzado regional no logra obtener la eficiencia adecuada debido a la falta de capacitación del personal involucrado en la fabricación de calzado, la carencia de tecnología adecuada para mejorar los procesos disminuyendo los desperdicios al producir y mejorando la calidad de los insumos, la falta de conocimiento de las normas técnicas de calidad, no cumplen con los niveles de estandarización internacionales, entre otros. García (2017) mencionó a la tecnología tradicional que usan los fabricantes de calzado, la falta de innovación y creatividad para sus productos y la falta de asociación para trabajar en equipo, son sus mayores dificultades para enfrentar la competencia mundial.

Realizando una exhaustiva revisión de los antecedentes internacionales, se encontró el aporte de los siguientes académicos como Borrayo, Mendoza, y Castañeda (2019) quienes sustentan que el crecimiento empresarial de la industria del calzado en diferentes partes del mundo se ve reflejada por el aporte de sus diferentes factores que contribuyen en el éxito de la micro y mediana empresa. Así mismo, Yulier y Romero (2018) mirando alrededor del mundo a los países como Australia y Nueva Zelandia, observan que las estrategias de innovación y competitividad se exponen con algún detalle como eje de la competitividad y crecimiento para las Mypes en esos espacios geográficos. Para Moguillansky, (2006)

Esta obra se comparte bajo la licencia Creative Common Atribución-No Comercial 4.0 International (CC BY-NC 4.0)

Revista de la Universidad Internacional del Ecuador. URL: https://www.uide.edu.ec/ 
considera que el análisis problemático de las Mypes en china se centra básicamente en factores tales como: aportación a la creación de empleo, comportamiento ante la innovación tecnológica, procesos estandarizados, barreras financieras y políticas, y medidas de gobierno.

Por otro lado, en América Latina, las organizaciones productivas diversificadas y los artículos de bajo valor agregado intervienen de manera decidida en el desempeño de las empresas e inciden en la baja exportación de productos de calzado. Así tenemos que para Reyes, Aldas, Morales y García (2016) sostienen en sus análisis, que la industria de calzado presenta un fuerte desafío comercial, considerando los diferentes factores de éxito de éste, los cuales no permiten continuar con el crecimiento empresarial, tales como desorden, desorganización, falta de control, problemas de financiamiento y sobre todo la pérdida de eficiencia en la pequeña y mediana empresa.

En el mismo contexto, se revisó las fuentes nacionales como Azañedo y Valverde (2015), y Hernando de Soto (2007), quienes afirman que existen factores que restringen el crecimiento del negocio de calzado en la región de La Libertad. La industria peruana de calzado es mínima, a lo que representa el mercado mundial. Uno de los retos para las empresas del sector calzado será invertir un poco más en el área de diseño, para ampliar el desarrollo de modelos propios para exportar sin necesidad de andar copiando algunas tendencias o modelos del exterior. De otro lado, Aranda (2015) menciona que la industria de calzado trujillano ha generado un dinamismo en toda la región de la Libertad. Más aun en las fechas con festividades tales como el inicio del año escolar o fiestas costumbristas de la región, las ventas se incrementan en un 30\%, logrando añadir alrededor de 300 millones de soles al mes a la economía liberteña.

El orden en el marco conceptual es que toda investigación debe tener una base teórica, que brinde el soporte necesario para sustentar las diferentes categorías o conceptualizaciones de estudio.

\section{Factores}

Según Okpara y Wynn (2007) las políticas y el gobierno son quienes ponen barreras para las Mypes, y éstas plantean una taxonomía basada en decisiones para empezar y conservar un negocio triunfante. Estas categorías se distribuyeron de la siguiente manera: (a) factores operativos, permiten examinar y dosificar los activos en una manera adecuada y adhieren aspectos semejantes en temas operativos, almacenes y de publicidad. (b) factores estratégicos, están las concernientes a la capacidad de los empresarios para tener un conocimiento a largo plazo, ajustar sus bienes y servicios con la proyección de la demanda, a escoger la mixtura de mercaderías que se promoverán y a segmentar a los clientes. (c) factores administrativos, hacen reseña al diseño organizacional y a la capacidad innata de lograr y desplegar los puntos a favor, imperiosos para la organización de forma tal que admitan extender el accionar positivo. Un fragmento en el inconveniente administrativo funciona en correlación directa a la distribución con los horizontes escalonados, los compromisos, las etapas de trabajo y comunicación, los conductos y los puntos de atención al cliente; y de otro lado hace referencia a la captación y selección del personal y su preparación, el avance con que abastecerán los materiales, el sistema crediticio y la compra de muebles y unidades, considerando los ítems de capitales y gerencia. (d) factores externos, llevan concordancia con la tecnología, la demanda y sus intermitentes, la corrupción a nivel general. 
Tomando en cuenta al crecimiento se revisó el aporte académico de Galvis (2016) y Meierkord (2016), quienes afirman que el crecimiento es el procedimiento por el cual la compañía crece de tamaño o dimensión, generalmente como efecto del aumento de la demanda. Esta definición establece una interrelación entre el desarrollo de la empresa y el tamaño del crecimiento. Asimismo, el objeto del desarrollo está estrechamente conectado con la función de utilidad de los dueños de la empresa.

\section{Mypes}

Como parte de la conceptualización de las variables de estudio, podemos plasmar la definición de las Mypes. E-Mypes (2019) refiere que las Mypes son unidades económicas que tiene como actividades principales, la extracción, producción, transformación, comercialización y creación de bienes y servicios, y según la Sunat (2019) éstas tienen las siguientes características paras ser consideradas como micro empresa: (a) número de trabajadores, de uno hasta diez trabajadores, y (b) ventas anuales, hasta un monto de 150 unidades impositivas tributarias. Con respecto a la pequeña empresa: (a) número de trabajadores, de uno hasta cien trabajadores, y (b) ventas anuales superiores a 150 unidades impositivas tributarias y hasta el monto máximo de 1,700 unidades impositivas tributarias. El monto de la unidad impositiva tributaria (UIT) para 2019 es de S/ 4,200 nuevos soles.

Según el aporte de Chiavenato (2006) la comprensión de las organizaciones se inicia identificando su característica principal, que es una clase de sistemas sociales. Ello significa precisar las etapas que sostienen las continuidades operacionales que les permiten mantener sus ciclos en medio de ambientes dinámicos. Spasić \& Kesić (2019) y Giordano (2015) sostienen que la teoría de sistema es un conjunto de objetos, elementos, sistemas, partes, componentes, procesos, que se interrelacionan para poder tener un propósito en común. Por lo que, para determinar el crecimiento de una organización, denominada micro o mediana empresa, está conformada por varias unidades y procesos que se desprenden de otras unidades, con el objetivo de sumar sinergias y poder evolucionar en el tiempo. También Hai (2017) afirma que el crecimiento empresarial, se puede llevar a cabo por diferentes factores que son la línea base para el crecimiento, debido a que son dependientes de otros procesos relacionales, por lo tanto, se evidencia el uso de la teoría de sistemas como base de la investigación.

En la teoría de las relaciones humanas, Panesso (2017) sostuvo que el ser humano requiere la interacción con otras personas, para tener una convivencia social, en ese mismo orden Chávez y Vizcaíno (2017) recalcan el valor del individuo y de las conexiones sociales en la vida organizada, sugieren implementar estrategias para elevar la satisfacción del integrante de ésta y alcanzar su potencial, teniendo en cuenta que los seres humanos constituyen el factor básico del éxito empresarial.

En este sentido, el presente estudio analiza tales factores, con el objetivo de incrementar sus probabilidades de éxito, disminuir las tasas de mortalidad de estas empresas y aumentar sus posibilidades de supervivencia en el largo plazo. Para ello, se formula el siguiente problema de investigación: ¿Cuáles son los factores que inciden en el crecimiento de la micro y pequeña empresa en la industria del calzado - Trujillo 2019?; los problemas específicos son: ¿Cómo los Factores administrativos inciden en el crecimiento de la micro y pequeña empresa en la industria del calzado - Trujillo 2019?, ¿Cómo los factores operativos inciden en el crecimiento de la micro y pequeña empresa en la industria del calzado - Trujillo 2019?, ¿Cómo los factores estratégicos inciden en el crecimiento de la micro y pequeña 
empresa en la industria del calzado - Trujillo 2019?, ¿Cómo los factores externos inciden en el crecimiento de la micro y pequeña empresa en la industria del calzado - Trujillo 2019 ?. Para la formulación del objetivo general, se plantea lo siguiente: analizar los factores que inciden en el crecimiento de la micro y pequeña empresa en la industria del calzado - Trujillo 2019, los objetivos específicos son: analizar los factores administrativos que inciden en el crecimiento de la micro y pequeña empresa en la industria del calzado - Trujillo 2019., analizar los factores operativos que inciden en el crecimiento de la micro y pequeña empresa en la industria del calzado - Trujillo 2019., Analizar los factores estratégicos que inciden en el crecimiento de la micro y pequeña empresa en la industria del calzado - Trujillo 2019.y analizar los factores externos que inciden en el crecimiento de la micro y pequeña empresa en la industria del calzado - Trujillo 2019.

Para la justificación teórica, el estudio se sustenta en la teoría de sistemas que es un conjunto de sistemas, que se interrelacionan entre sí para poder tener un propósito en común y la teoría de las relaciones humanas donde se recalca el valor del individuo y de las conexiones sociales en la vida organizada, al elevar la satisfacción del integrante de ésta y alcanzar su potencial. Sitharam \& Hoque (2016) permitirá ver el trabajo, qué elementos son determinantes para el crecimiento de las Mypes. En el rubro de calzado, si es posible como nación tender a especializarse con un precio relativamente más pequeño frente al resto del mundo, o diferenciándonos por su diseño y calidad, creando más fuentes de trabajo, más pago de tributos y por ende mayor desarrollo de la nación.

Para la justificación metodológica el presente trabajo ha afianzado la elaboración de cuatro factores que determinan el crecimiento de las mypes de calzado en la ciudad de Trujillo y su aplicación mediante entrevistas en profundidad para cada una de los factores, situaciones que pueden ser investigadas por la ciencia, una vez que sean demostradas su confiabilidad podrán ser utilizados en otros trabajos de investigación.

Para la justificación práctica, este trabajo va a beneficiar a tres partes involucradas durante el ciclo productivo de la industria del calzado, entre estos tenemos: (a) beneficio directo a las Mypes, porque les va a permitir tener información real de los factores que miden el crecimiento económico; (b) beneficio a los empleados, porque el crecimiento empresarial del sector va a permitir la elaboración de nuevos puestos laborales, el cual será mediante mano de obra técnica; y (c) beneficio al gobierno, al fomentar a través del empleo, la mejora de los procedimientos de productividad y mayores ingresos tributarios.

\section{Método}

Según Hernandez \& Mendoza (2018) y Hernández, Fernández \& Baptista (2014) sostienen que un enfoque cualitativo tiene un análisis profundo de forma subjetiva; que permitirá profundizar los fenómenos, explorándolos desde la perspectiva de los participantes en su espacio laboral y en relación con el contexto. El método de investigación que se aplicará en el presente artículo es el estudio de caso que se necesita comprender la situación y significado de los empresarios a ser entrevistados. Los estudios de casos son descripciones de sistemas simples tales como un individuo, un programa, un grupo o una comunidad. Marradi, Archenti, \& Piovani (2007).

En este sentido, Díaz (2018) expone que dichos procesos son la fase de la formulación del problema de investigación; fase de planeamiento donde aparece la importancia de la 
investigación, su estrategia metodológica y escritura del proyecto; fase de entrada, donde aparecen los informantes y la aplicación de los instrumentos; fase productiva y análisis preliminar; fase de salida de campo y análisis extenso; y por último la fase de escritura. Por otro lado, se debe considerar que hay decisiones las cuales están fuertemente influenciadas por factores éticos e ideológicos, contenidos para configurar el problema de investigación.

Tabla 1.

Matriz de categorización: factores de crecimiento

\begin{tabular}{ll}
\hline Sub categorías & Indicadores \\
\hline Factores Administrativos & Recursos humanos \\
& Finanzas \\
Factores Operativos & Administración general \\
& Marketing \\
Factores Estratégicos & Logística \\
& Operaciones \\
Factores Externos & Planeamiento \\
& Investigación de mercados \\
& Infraestructura \\
& Informalidad \\
\hline
\end{tabular}

Fuente: Elaboración propia

Divakaran (2014) sostiene que la investigación se encuentra interrelacionado y que tiene cierta particularidad para responder el estudio. El presente análisis corresponde a 12 casos de empresarios de la pequeña y microempresa de la industria del calzado y 03 personas especialistas en el rubro de calzado, que será desarrollado en el segundo semestre de 2019. El lugar de su aplicación corresponde a la región de La Libertad (Trujillo), específicamente en el distrito de El Porvenir, donde se concentra la mayoría de micro y pequeños empresarios del rubro de calzado, lugar que se destaca por que la mayoría de su gente actúa directa o indirectamente en el negocio de calzado. En El Porvenir las pequeñas y micro empresas de calzado son en un $98 \%$ familiares. Trabajan alrededor de tres mil familias dedicadas a la fabricación de zapatos, con más de 30 años en el mercado del calzado.

\section{Resultados}

\section{Análisis descriptivo Características generales del propietario-gerente de la empresa}

Considerando los resultados del grafico 1, en relación a las características resaltantes de los propietarios de las empresas analizadas son: el $80 \%$ de los mismos son de género masculino, $75 \%$ tienen más de cinco años de experiencia en el negocio y afirman no ser conservadores. Por otro lado, el $67 \%$ cuenta con estudios técnicos y universitarios. 


\section{Gráfico 1}

Formación de los propietarios de las empresas

\section{FORMACION ACADEMICA}

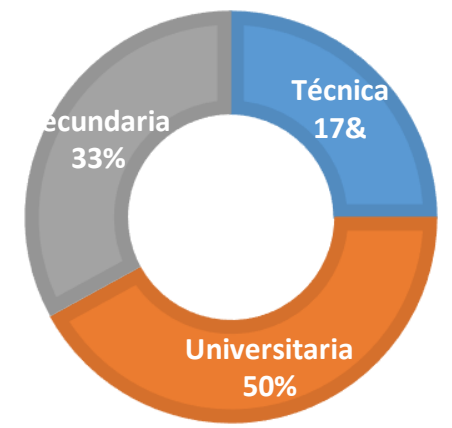

Fuente: Elaboración propia

Estos resultados obtenidos guardan cierta concordancia con el estudio realizado por OSEL Lima Norte (2014), en el cual se encontró que en el sector calzado el 41,4\% de los empresarios cuenta con estudios universitarios y en Trujillo en el sector calzado hay un 50\% de empresarios con estudios superiores. El principal motivo que les impulsó a emprender fue mejorar su nivel económico y el deseo de éxito personal, que de forma natural conduce la estrategia de crecimiento de la organización (Child y Keiser, 1981).

\section{Características generales de las empresas}

La característica que destaca en las empresas analizadas es que el 75\% de las mismas tienen más de 10 años de experiencia en el mercado, lo cual en muchos de los casos es un acicate para los negocios. Es decir, a mayor tiempo en una actividad, genera habilidades y apoyan favorablemente al crecimiento de la empresa (Arregle et al., 2013).

\section{Gráfico 2}

Años de funcionamiento de la empresa

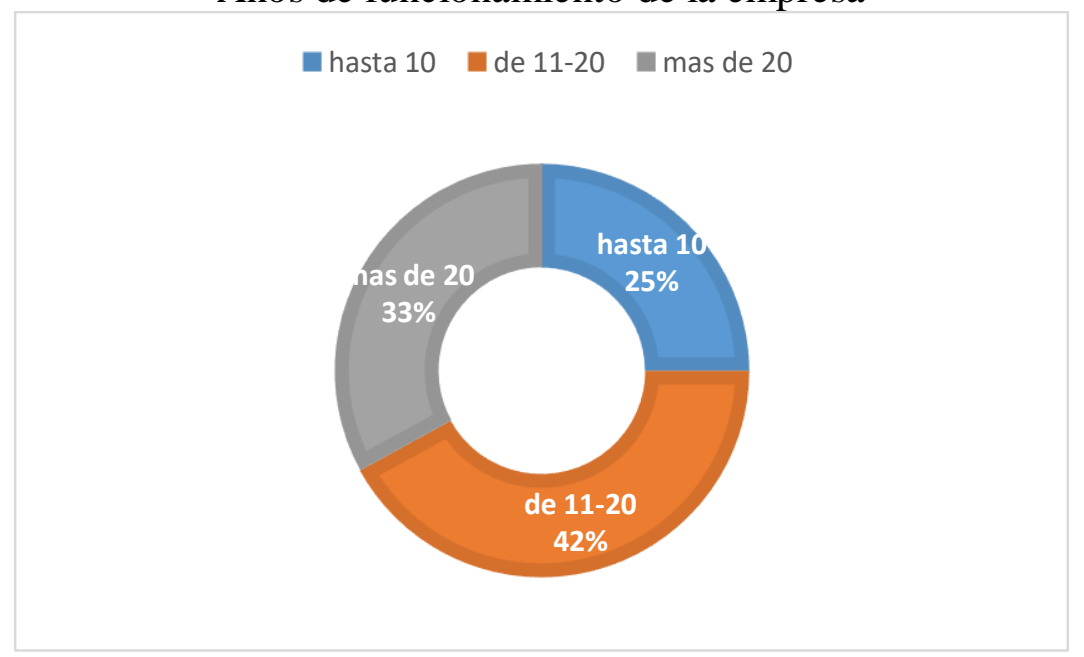

Fuente: Elaboración propia 
Asimismo, las empresas analizadas presentan una antigüedad promedio de 16,83 años de funcionamiento, lo que demuestra una acumulación de experiencia y establecimiento de rutinas que favorecen la eficiencia y el crecimiento de los negocios (Sánchez y García, 2003).

\section{Resultados de las entrevistas en profundidad}

El trabajo de investigación ha permitido identificar los factores que tienen incidencia con el crecimiento de las mypes de calzado en el Perú.

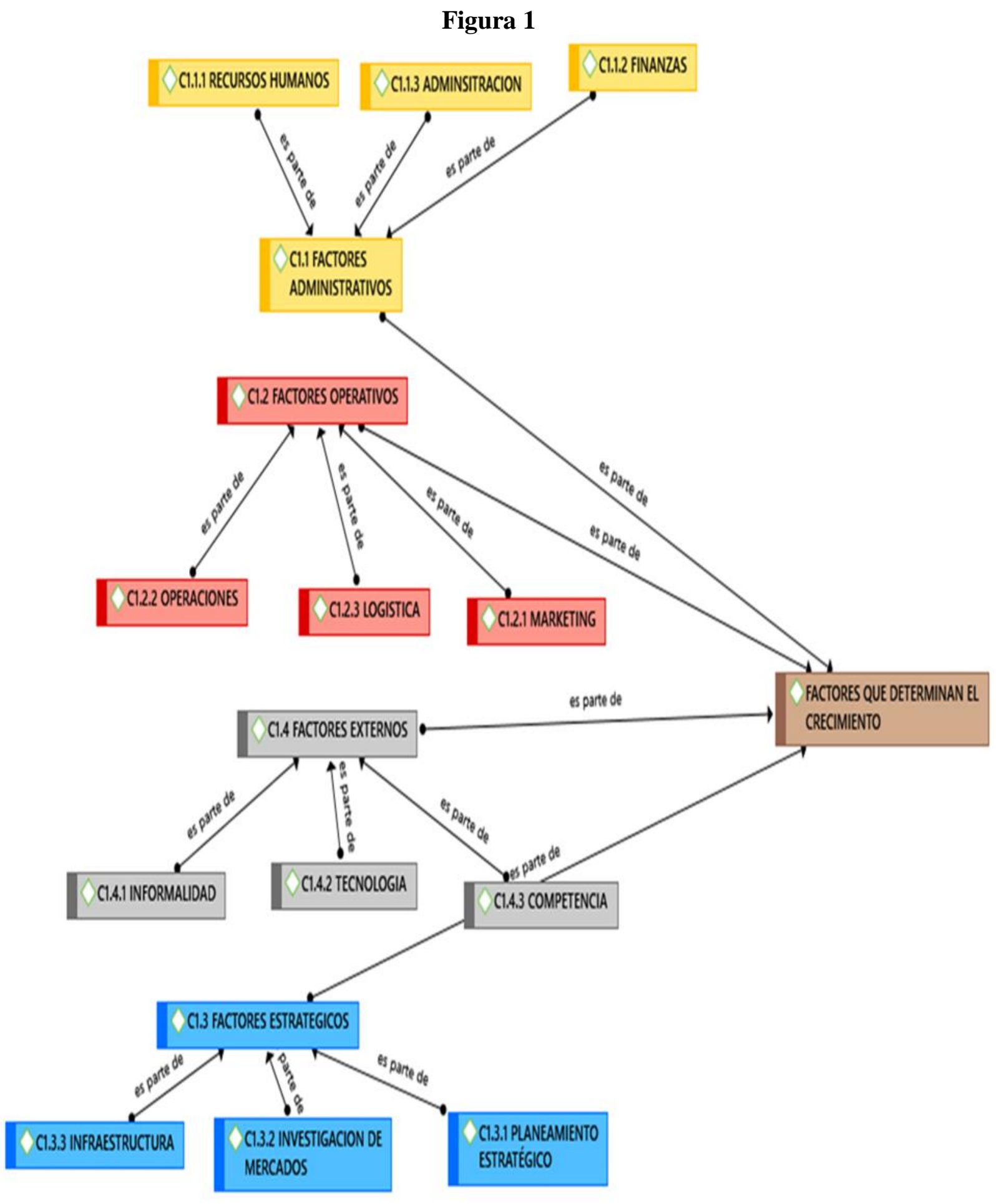

Fuente: Elaboración propia

Esta obra se comparte bajo la licencia Creative Common Atribución-No Comercial 4.0 International (CC BY-NC 4.0) Revista de la Universidad Internacional del Ecuador. URL: https://www.uide.edu.ec/ 
Las micro y pequeñas empresas del sector calzado estudiadas han determinado que los factores que tienen una mayor incidencia para su crecimiento empresarial son los que se presentan a continuación

\section{Gráfico 3}

Factores que determinan el crecimiento del sector calzado

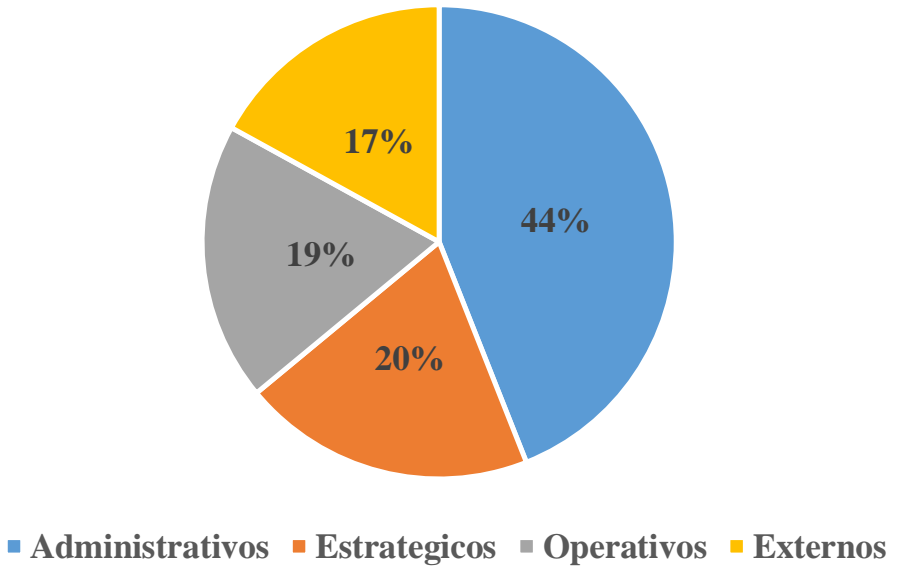

Fuente: Elaboración propia

Según Blázquez, Dorta y Verona (2006) argumentaron que el crecimiento empresarial depende de diversos factores que pueden ser internos o externos. Los empresarios del sector calzado de Trujillo deben realizar fuertemente mejoras en el campo de los factores administrativos, ya que una buena administración del negocio tiene que ver con la parte operativa de como producir los diversos productos de calzado, el área de marketing, la gestión contable financiera, el buen uso de la tecnología y las redes sociales, la gestión de personas y buenas decisiones estratégicas conducen siempre a mejores resultados en el tiempo.

Con respecto a los factores administrativos, que tiene que ver con la gestión empresarial, el estudio permitió arribar a las a las siguientes conclusiones:

\section{Gráfico 4}

Factores Administrativos

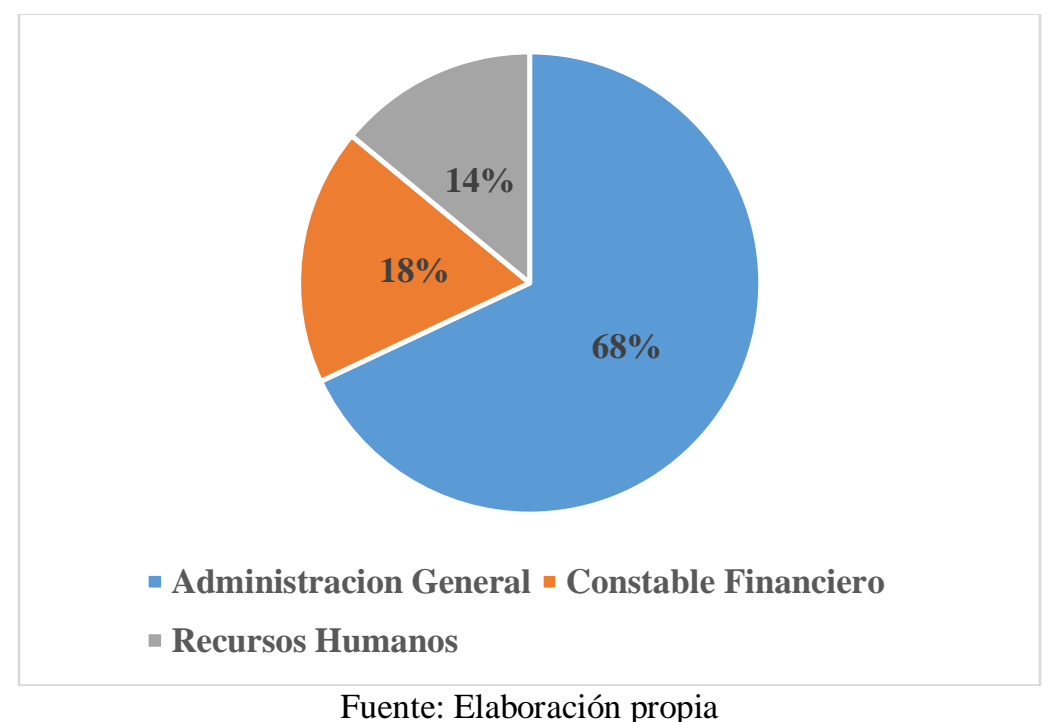

Fuente: Elaboración propia 
En el grafico se puede visualizar que los micro y pequeños empresarios del sector calzado de la ciudad de Trujillo manifiestan que, dentro de los factores administrativos, el factor que más limita su crecimiento es el de la administración general con un $68 \%$, ya que no le ayuda para alcanzar sus objetivos empresariales. Abarca desde generar qué tipo de marca debe llevar la empresa, pasando por desarrollar estrategias de marketing para satisfacer a los diversos clientes que tiene la empresa, la etapa de los productos que necesitan los clientes, como los quieren, la calidad de los insumos, y los precios que estos demandan, Los aspectos contables financieros le siguen en orden descendente con un $18 \%$ y son necesarios para conocer la situación de la empresa y continua los recursos humanos con un 14\%, que generalmente se les capacita en la etapa inicial cuando es incorporado a la empresa Con respecto a los factores operativos, que muestra el proceso productivo del sector calzado el estudio permitió arribar a las a las siguientes conclusiones:

Gráfico 5

Factores Operativos

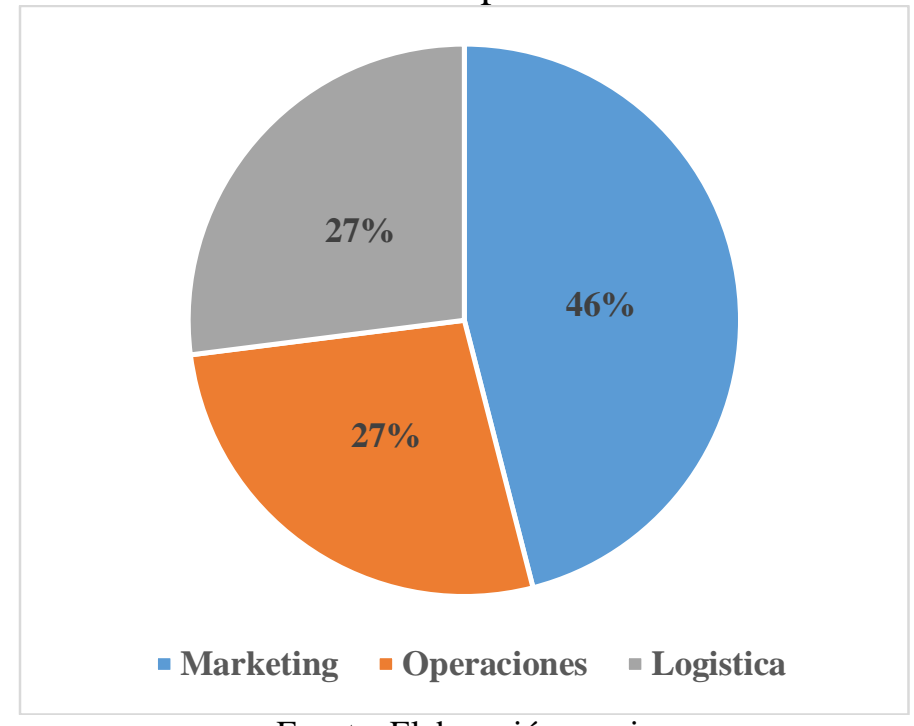

Fuente: Elaboración propia

Los empresarios manifiestan que se debe dar un mayor impulso al marketing para alcanzar sus objetivos empresariales, presentando productos mejorados en diseños innovadores y calidad de insumos, que les permitan permanecer en el mercado. "Lo que siempre busco es identificar cosas nuevas que ofrecer" (Entrevistado 4). "La competencia es fuerte, yo vivo con la experiencia, y todo lo que estoy haciendo es gracias a mi esfuerzo" (Entrevistado 5). Esto es congruente con el factor operativo y el de logística que tienen un peso igual en concordancia para los empresarios y en relación con el control de inventarios, la mayoría de los empresarios entrevistados posee conocimientos básicos; y lo llevan en un programa de Excel, generando el registro de los movimientos de entrada y salida "En realidad, nuestro control es bien practico, lo llevamos nosotros mismos, sabemos cuánto material tenemos en el almacén y cuánto vamos despachando" (Entrevistado 2).

Con respecto a los factores estratégicos, con el que los empresarios se basan para alcanzar sus metas y sueños empresariales, el estudio permitió arribar a las a las siguientes conclusiones: 


\section{Gráfico 6}

Factores Estratégicos

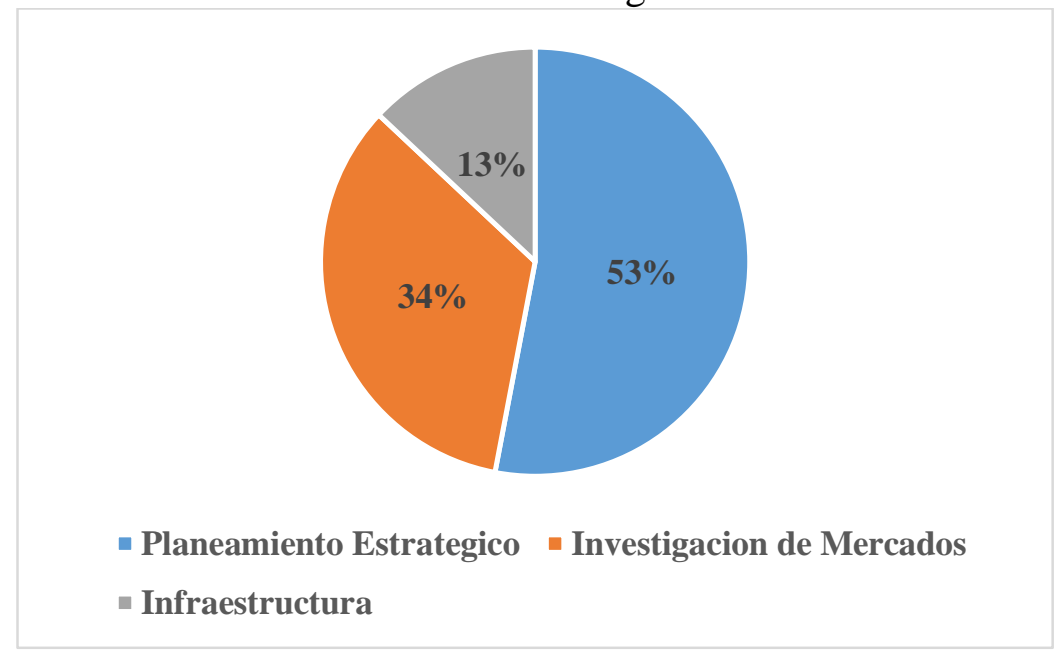

Fuente: Elaboración propia

Con respecto a los factores estratégicos, los micro y pequeños empresarios del sector calzado de la ciudad de Trujillo opinan que el factor más limitante para su crecimiento es el factor planeamiento estratégico con un 53\%, y lo relacionan con la administración general, consolidando un factor de gerencia. Le sigue en orden de prioridad la investigación de mercados con un 34\%, y es consistente con la innovación de los nuevos modelos, los colores, los insumos y como es que desean los clientes los productos a desarrollar, y en el siguiente orden está la infraestructura con un $13 \%$ y está relacionado con la posibilidad de contar con activos fijos para trabajar con más tranquilidad y poder aspirar a exportar con mayor eficiencia.

Con respecto a los factores externos, que nos permite mirar fuera de la organización, el estudio permitió arribar a las siguientes conclusiones:

\section{Gráfico 7}

Factores Externos

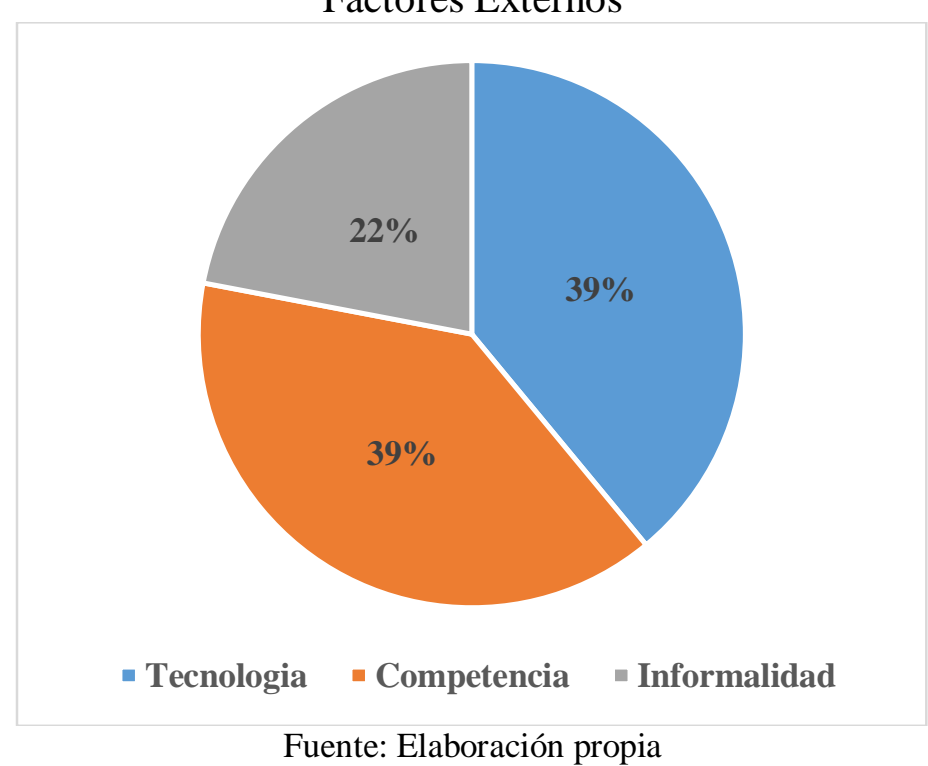

Fuente: Elaboración propia 
Con respecto a los factores externos se puede afirmar que los micro y pequeños empresarios del sector calzado de Trujillo opinan que los factores que más limitan su crecimiento están relacionados con la competencia con un $39 \%$, donde se manifiesta la competencia desleal, desde el punto de vista de la informalidad o del dumping. Continúa en orden de afinidad a la competencia el factor informalidad con un $22 \%$, relacionado con no estar constituida legalmente y no contribuir con sus impuestos al estado peruano, y el factor tecnología con un 39\%, que está relacionado directamente con el uso de redes sociales, estar al tanto de lo novedoso en el sector calzado y el uso eficiente de la tienda virtual.

\section{Conclusiones}

Las micro y pequeñas empresas de la industria del sector calzado estudiadas pertenecen a las empresas formales y actúan de acuerdo con la ley General de Sociedades. Las limitaciones que presentan estas empresas para alcanzar su crecimiento empresarial se deben básicamente a o factores de la gestión empresarial.

Dentro de los factores administrativos que limitan el crecimiento de las mypes de calzado, los temas identificados más importantes tienen relación con la administración propia de sus negocios. Los empresarios del sector calzado deben realizan fuertemente mejoras en el campo de la administración en general, a partir de su conocimiento operativo, una buena gestión de personas y de decisiones estratégicas conducen a mejores resultados en el tiempo.

En relación con los factores de carácter operativo que limitan el crecimiento de las mypes de calzado, para poder competir y mantenerse en el mercado, los empresarios consideran que deben desarrollan más estrategias relacionadas con él marketing, calidad de los productos y precios justos para generar un posicionamiento en el mercado. En menor escala, en logística conocimientos básicos respecto a las operaciones de producción y el control de inventarios, la relación con los proveedores se basa en un nivel de confianza y calidad.

En cuanto a los factores de carácter estratégico, los empresarios tienen muy claro en desarrollar planes estratégicos que les sirvan de guía para la gestión de sus empresas, además, no existe un patrón definido que haya permitido el crecimiento de las mypes de calzado. Los empresarios están de acuerdo que el planeamiento estratégico es esencial para consolidar a la organización en un lugar expectante del sector y así permitir lograr su crecimiento empresarial.

Respecto de los factores externos, la tecnología y la competencia son las barreras que limitan más a estas empresas su crecimiento y un poco menos la informalidad. El rol del Estado es insuficiente, al no desarrollar mecanismos y programas que promuevan la formalización y capacitación.

El crecimiento empresarial en el Perú es un tema complejo, involucra múltiples dimensiones: política, social, cultural, ideológica y económica que, si no se aborda de una manera articulada, desde la academia, las empresas y el estado, las posibilidades de crecimiento y desarrollo del país serán limitadas. 


\section{Recomendaciones}

Fortalecer la actuación gerencial de los empresarios de las mypes del sector calzado de Trujillo para lograr su crecimiento, por lo que se sugiere promover talleres, seminarios, cursos y programas de capacitación integral con apoyo de CITEccal.

Generar un mecanismo adecuado que permita a las mypes de calzado acceder a préstamos con menos burocracia en las diferentes entidades financieras y con tasas de interés justas tanto para capital de trabajo como para activos fijos, que fortalezcan su crecimiento empresarial.

Permitir acceder a la tecnología apropiada por parte de las mypes del sector calzado de Trujillo en los diferentes procesos de producción puede colaborar con su crecimiento, ayuda a reducir costos en el proceso productivo y estar acorde con el avance de la moda a nivel global.

Obtener mecanismos en coordinación con un Estado más proactivo y dinámico que colabore para que las mypes de calzado puedan operar dentro de la formalidad ampliando la base tributaria, lograr una competencia más equilibrada en el sector y no generar más distorsión en el mercado de calzado.

\section{Bibliografía}

Aguirre, J. y. (2015). El papel de la descripción en la investigación cualitativ. Scielo, pp 175189.

AMPEX. (12 de Mayo de 2018). http://www.peruchamber.com. Obtenido de www.peruchamber.com: http://www.peruchamber.com/stdwww.cfm?reg_id=14\&org_id=106\&x1=Mx46d

Anderson, D., Sweeney, D., \& Williams, T. (2015). Essentials of Modern Business Statistics with Microsoft Excel. Boston: Cengage Learning.

Aranda, W. (03 de 10 de 2015). Industria del calzado Trujillano. La republica.

Avolio, B., Mesones, A., \& Roca, E. (2007). Factores que Limitan el Crecimiento de las Micro y Pequeñas Empresas en el Perú (MYPES). Lima: Centrum Católica.

Azañedo Roldan, M. y. (2015). Oportunidad de negocio en el mercado canadiense para la exportación de calzado de la empresa Calzados Kattia EIRL de la Region La Libertad, año 2015. La Libertad.

Blazquez, F., Dorta, J., \& Verona, M. (2006). Factores del crecimiento empresarial. Innovar.

Borrayo, R., Mendoza, M., \& Castañeda, M. (2019). Productividad y eficiencia técnica de la industria manufacturera regional de México, 1960-2013: un enfoque panel de frontera estocástica. Estudios Económicos, 25 - 60.

Caruajulca, S., \& Férnandez, K. (2016). Plan estratégico para la creación de valor en una empresa de fabricación de calzado en la libertad - 2016. Trujillo: Universidad Nacional de Trujillo.

Castelló, I. (2017). Análisis del sector del calzado en la Ciudad Valenciana y estudio de propuesta de internacionalización. Valencia: Universidad Politécnica de Valencia.

Esta obra se comparte bajo la licencia Creative Common Atribución-No Comercial 4.0 International (CC BY-NC 4.0)

Revista de la Universidad Internacional del Ecuador. URL: https://www.uide.edu.ec/ 
chavez, E., \& vizcaino, A. (04 de 2017). Talento Humano; una contribucion a la competitividad organizacional. Mercados y Negocios, 07 - 20.

Chiavenato, I. (2006). Introducción a la teoría general de la administración. Distrito Federal: McGraw-Hill Interamericana.

Ciceg. (29 de Marzo de 2019). http://www.ciceg.org. Obtenido de www.ciceg.org: http://www.ciceg.org/boletines/editorial_luis_gerardo/edit14.pdf

Creswell, J. (2005). Educational research: Planning, conducting, and evaluating quantitative and qualitative research. liberabit.

Diaz, C. (2018). Investigación cualitativa y análisis de contenido temático. Revista General de Información y Documentación.

Divakaran, P. (2014). Research Methodology. Bhopal: Horizon Books.

Dzul, M. (19 de Enero de 2019). www.uaeh.edu.mx. Obtenido de https://www.uaeh.edu.mx: https://www.uaeh.edu.mx/docencia/VI_Presentaciones/licenciatura_en_mercadotecni a/fundamentos_de_metodologia_investigacion/PRES39.pdf

E-mypes. (2 de Julio de 2019). http://www.e-mypes.com. Obtenido de www.e-mypes.com: http://www.e-mypes.com/comercio-exterior/que-es-una-mype.php

Flores, J., \& Jiménez, L. (2017). El sector del calzado a nivel internacional y los factores criticos de éxito. Distrito Federal: Pegasus.

Galvis , Y. (2016). Comunicación: reputación y crecimiento empresarial. Pensamiento y gestión, 46.

Garcia Ramis, e. v. (2017). creatividad e innovacion en los equipos d etrabajo. linkedin.

Garrell, A. (2019). La Industria 4.0 en la sociedad digital. Barcelona: Marge Books.

Gendron, L., \& Plourde, L. (2010). Los factores del éxito de las pymes del Saguenay y que se desempeñan en el sector del aluminio. scielo.

Giordano, P. M. (2015). Avatares del cambio en la Teoría General de la Acción de Talcott Parsons. Scielo.

Gonzalo, M., Federico, J., \& Kantis, H. (2013). Crecimiento y adaptación en un contexto de crisis internacional: . Programa de Desarrollo Emprendedor .

Hai, S. (2017). Data Analytics in Digital Humanities. Manhattan: Springer.

Hernandez, R., \& Mendoza, C. (2018). Metodología de la investigación. Las rutas cuantitativa, cualitativa y mixta. Mexico: Mc Graw Hill Education.

Hernández, R., Fernández, C., \& Baptista, P. (2014). Metodología de la Investigación. Distrito Federal: McGraw-Hill.

icex. (2017). Elmmercado de calzado en japom. España: Ofiicna comercial de españa en tokto

Marradi, A., Archenti, N., \& Piovani, J. (2007). METODOLOGÍA DE LAS CIENCIAS SOCIALES. Argentina: Grupo Interuniversitario Postdata. 
Mosquera, F., Rojas, S., \& Robayo, O. (2017). Orientación al Mercado en las Pequeñas Empresas del Sector Calzado en Cuero de la Ciudad de Bogotá. Redalyc, 17.

Muñiz, E. (2008). La investigación "Factores de éxito y su relación con el Crecimiento Empresarial,. Economía y organización de la empresa.

Okpara, J., \& Wynn, P. (2007). SAM Advanced Management Journal. NewYork: Journal.

Panesso, K. y. (2017). La Autoestima, Proceso humano. Medellin, Colombia: Universidad de Antioquia.

Reyes, J., Aldas, D., Morales, L., \& García, M. (2016). Evaluación de la capacidad para montaje en la industria manufacturera de calzado. Ingeniería Industrial - Universidad Técnica de Ambato, 10.

Samaja, J. (2018). Methodological triangulation (for a dialectic understanding of the approaches combination). Scielo.

Serida, J., Alzamora, J., Guerrero, C., Borda, A., \& Morales, O. (2016). Global Entrepreneurship Monitor: Perú 2015-2016. Lima: Universidad ESAN.

Sunat. (11 de Julio de 2019). www.sunat.gob.pe. Obtenido de http://emprender.sunat.gob.pe: http://emprender.sunat.gob.pe/que-beneficios-tengo

Urcia, M. (2013). Capacidad de innovación empresarial de las micro y pequeñas empresas de calzado del distrito El Porvenir, Trujillo, Perú. Revista "Ciencia y Tecnología”, 17.

Yulier, S., \& Romero, J. (2018). Revisión del estado actual de la industria de las curtiembres en sus procesos y productos: un análisis de su competitividad. Revista Facultad de Ciencias Económicas, 12. doi:DOI: https://doi.org/10.18359/rfce.2357 